\title{
ON AN ANALOGUE OF THE JAMES CONJECTURE
}

\author{
GEORDIE WILLIAMSON
}

Dedicated to Jimi

\begin{abstract}
We give a counterexample to the most optimistic analogue (due to Kleshchev and Ram) of the James conjecture for Khovanov-Lauda-Rouquier algebras associated to simply-laced Dynkin diagrams. The first counterexample occurs in type $A_{5}$ for $p=2$ and involves the same singularity used by Kashiwara and Saito to show the reducibility of the characteristic variety of an intersection cohomology $D$-module on a quiver variety. Using recent results of Polo one can give counterexamples in type $A$ in all characteristics.
\end{abstract}

\section{INTRODUCTION}

A basic question in representation theory asks for the dimensions of the simple modules for the symmetric group over an arbitrary field. Despite over a century's effort even a conjectural formula remains out of reach.

Any simple representation in characteristic zero may be represented by integral matrices, and one obtains a representation in characteristic $p$ by reducing these matrices modulo $p$. This induces a well-defined map on Grothendieck groups, and the matrix of this map (in the basis of simple modules) is the "decomposition matrix". Knowledge of the decomposition matrices for the symmetric group would give a formula for the dimensions of the simple modules in characteristic $p$.

The group algebra of the symmetric group has a deformation given by the Hecke algebra, and if one specialises the quantum parameter to a $p^{\text {th }}$-root of unity one obtains an algebra in characteristic zero whose representation theory is similar to that of the symmetric group in characteristic $p$. The Hecke algebra allows one to factor the decomposition matrix as the product of two matrices: the first controls specialising the quantum parameter (a characteristic zero question), and the second controls reduction modulo $p$. Moreover, in 1990 James [J] conjectured that this second "adjustment" matrix should (under certain explicit lower bounds on the characteristic) be trivial 1

The first matrix (controlling the characters of simple modules for the Hecke algebra at a $p^{\text {th }}$ root of unity) may be calculated using Schur-Weyl duality and Lusztig's character formula for quantum groups at a root of unity [L1]. More recently, Lascoux, Leclerc and Thibon [LLT] formulated conjectures giving a faster algorithm to calculate these matrices in terms of the dual canonical basis of Fock space for the quantum affine special linear group. These conjectures were subsequently proved by Ariki $[\mathrm{A}$. (A proof was also announced by Grojnowski.) Hence the Hecke algebra

Received by the editors April 4, 2013 and, in revised form, May 10, 2013 and October 2, 2013. 2010 Mathematics Subject Classification. Primary 20C08, 20C20, 20 C30.

${ }^{1}$ After this article was submitted the author discovered a way of producing counterexamples to Lusztig's conjecture. These results may be used to deduce counterexamples to the James conjecture [W]. 
problem has been solved and one would like to know when the James conjecture is valid.

Over the last decade these ideas have been gathered under the umbrella of categorification and the important role played by Khovanov-Lauda-Rouquier (KLR) algebras [KL, R1] has become clear. This is mainly thanks to the Brundan-Kleshchev isomorphism [BK1]: the group algebras of symmetric groups, as well as Hecke algebras at roots of unity are isomorphic to cyclotomic KLR algebras. It follows that the representation theory of symmetric groups and Hecke algebras acquires a non-trivial grading. (The reader is referred to $[\mathrm{K}]$ for a survey of these and related ideas.)

The Brundan-Kleshchev isomorphism identifies the symmetric group algebra in characteristic $p$ and Hecke algebras at a $p^{t h}$ root of unity with KLR algebras associated to a cyclic Dynkin quiver with $p$ nodes (considered over a field of characteristic $p$ and 0 respectively). Using this isomorphism the James conjecture may be translated into the natural statement that the decomposition numbers for these KLR algebras are trivial, under certain explicit lower bounds on $p$.

The upshot is that KLR algebras associated to cyclic quivers could help us make progress on one of the oldest questions in representation theory. This begs the question: What do KLR algebras associated to Dynkin quivers mean? One might hope that their modular representation theory is simpler, and that we can garner a clue as to how to approach the cyclic case. The following conjecture was proposed by Kleshchev and Ram [KR, Conjecture 7.3] as a finite analogue of the James conjecture:

Conjecture 1.1. The decomposition numbers for KLR algebras associated to Dynkin quivers are trivial.

The purpose of this paper is to explain what this conjecture means geometrically, and give a counterexample. Over a field of characteristic zero it is a theorem of Varagnolo and Vasserot [VV] and Rouquier [R2] that KLR algebras are the Ext algebras of Lusztig sheaves on the moduli space of quiver representations. Hence understanding projective modules over KLR algebras is the same as understanding the decomposition of Lusztig sheaves with coefficients of characteristic zero. The Decomposition Theorem [BBD] allows one to translate this into a combinatorial problem. Maksimau [M] has recently extended this result: KLR algebras are the Ext algebras of Lusztig sheaves over $\mathbb{Z}$. Here the decomposition theorem is missing, but Kleshchev and Ram's conjecture is equivalent to the statement that the decomposition of Lusztig sheaves should remain the same with coefficients in any field.

This in turn is equivalent to asking that the stalks and costalks of intersection cohomology complexes on moduli spaces of quiver representations be free of torsion. (Or equivalently, that parity sheaves (see $\$ 3.6$ ) are isomorphic to intersection cohomology complexes.) A number of people had hopes that similar statements would be true on flag varieties in type $A$. In fact, the statement for moduli spaces of quiver representations would imply the corresponding statement for flag varieties, as we will see.2

In 2004 Braden gave counterexamples to these hopes on the flag variety of $G L_{8}$ WB. In this paper we explain the relation between these questions and give the

\footnotetext{
${ }^{2}$ It is possible that these two statements are equivalent: see the two sentences after Problem 1 in [KS §6.2].
} 
first counterexample to Conjecture 1.1. It occurs for the Dynkin quiver of type $A_{5}$ with coefficients of characteristic 2 . We also explain how one can use recent results of Polo to give counterexamples for type $A$ Dynkin quivers in all characteristics. Unfortunately, these examples are large: to get a counterexample in characteristic $p$ using the approach that we discuss here one needs to consider a KLR algebra associate to a Dykin quiver of type $A_{8 p-1}$ and dimension vector $(1,2, \ldots, 4 p, \ldots, 2,1)$. Hence the prospects of simple-minded attempts to use KLR algebras to actually understand what is going on seem a little dim!

Finally, the first counterexample (on the $A_{5}$ quiver in characteristic 2) is related to a certain subvariety in the moduli space of quiver representations which was used by Kashiwara and Saito to give a counterexample to a conjecture of Kazhdan and Lusztig on the irreducibility of the characteristic cycle [KS]. In fact, the examples that we discuss in this paper all have reducible characteristic cycles, by a result of Vilonen and the author [W]. Hence Kleshchev and Ram's conjecture would have been implied by Kazhdan and Lusztig's, had it been correct.

\section{BRAUER RECIPROCITY FOR GRADED RINGS}

In the modular representation theory of finite groups an important role is played by Brauer reciprocity. Brauer reciprocity is an equivalence between the two problems of determining the composition factors of the reduction modulo $p$ of a simple module in characteristic 0 , and decomposing the lift of a projective module in characteristic $p$ to characteristic 0 . It will be important for us because it rephrases the question of decomposition numbers for simple modules over KLR algebras into questions about projective modules. As we will see, the question of decomposing lifts of projective modules to characteristic 0 has a straightforward geometric interpretation.

2.1. Graded algebras and modules. Throughout we fix a prime number $p$ and let $\mathbb{F}=\mathbb{F}_{p}, \mathbb{O}=\mathbb{Z}_{p}$ and $\mathbb{K}=\mathbb{Q}_{p}$ (the finite field with $p$ elements, the $p$-adic integers, and the $p$-adic numbers respectively). All of the results of this paper remain valid for any $p$-modular system $(\mathbb{K}, \mathbb{O}, \mathbb{F})$.

Now let $H_{\mathbb{O}}=\bigoplus_{i \in \mathbb{Z}} H_{i}$ denote a finitely generated graded $\mathbb{O}$-algebra, such that $H^{i}$ is a finitely generated free $\mathbb{O}$-module for all $i \in \mathbb{Z}$. We set $H_{\mathbb{F}}:=\mathbb{F} \otimes_{\mathbb{O}} H$ and $H_{\mathbb{K}}:=\mathbb{K} \otimes_{\mathbb{O}} H$. These are also finitely generated graded algebras, finite-dimensional in each degree. We assume in addition that there exists a graded polynomial subring $A_{\mathbb{O}}=\mathbb{O}\left[X_{1}, \ldots, X_{m}\right] \subset H_{\mathbb{O}}$ with generators of positive degree such that:

(1) $A_{\mathbb{O}}$ is contained in the centre of $H_{\mathbb{O}}$;

(2) $A_{\mathbb{O}}$ is a summand of $H_{\mathbb{O}}$ as an $\mathbb{O}$-module;

(3) $H_{\mathbb{O}}$ is a finitely generated module over $A_{\mathbb{O}}$.

For $k \in\{\mathbb{F}, \mathbb{K}\}$ we set $A_{k}:=A_{\mathbb{O}} \otimes_{\mathbb{O}} k$ which is also a graded polynomial ring contained in the centre of $H_{k}$. Write $A_{k}^{+}$for the ideal of polynomials of positive degree. Any graded simple $H_{k}$-module is annihilated by $A_{k}^{+}$(by the graded Nakayama lemma) and hence is a module over $H_{k} /\left(A_{k}^{+}\right) H_{k}$, a finite dimensional graded algebra by our assumption (3). We conclude that $H_{k}$ has finitely many graded simple modules up to shifts, all of which are finite dimensional.

For $k \in\{\mathbb{K}, \mathbb{O}, \mathbb{R}\}$ let $\operatorname{Rep} H_{k}$ denote the abelian category of all finitely generated graded $H_{k}$-modules. That is, objects of Rep $H_{k}$ are graded left $H_{k}$-modules, and morphisms are homogenous of degree zero. Let $\operatorname{Rep}^{f} H_{k}$ denote the full subcategory 
of modules which are finite-dimensional over $k$, and let Proj $H_{k}$ denote the full subcategory of projective modules which are finitely generated over $H_{k}$. Given $M=\bigoplus M^{i} \in \operatorname{Rep} H_{k}$ let $M(j)$ denote its shift, given by $M(j)^{i}:=M^{j+i}$. Given $M, N \in \operatorname{Rep} H_{k}$ set $\operatorname{Hom}^{\bullet}(M, N):=\bigoplus \operatorname{Hom}(M, N(i))$. In this paper a graded category will always mean a category equipped with a self-equivalence (see the end of [R1, §2.2.1] for a discussion). We view Rep $H_{k}$ as a graded category with respect to the self-equivalence $M \mapsto M(1)$.

2.2. Grothendieck groups. The Grothendieck groups of $\operatorname{Rep}^{f} H_{k}$ and Proj $H_{k}$ will be denoted by $\left[\operatorname{Rep}^{f} H_{k}\right]$ and [Proj $\left.H_{k}\right]$ respectively. They are naturally $\mathbb{Z}\left[v^{ \pm 1}\right]$ modules by declaring $v[M]:=[M(1)]$. Given $P \in \operatorname{Proj} H_{k}$ and $M \in \operatorname{Rep}^{f} H_{k}$ we obtain a pairing $\langle-,-\rangle:\left[\operatorname{Proj} H_{k}\right] \times\left[\operatorname{Rep}^{f} H_{k}\right] \rightarrow \mathbb{Z}\left[v^{ \pm 1}\right]$ by setting

$$
\langle[P],[M]\rangle:=\underline{\operatorname{dim}} \operatorname{Hom}^{\bullet}(P, M) ;
$$

here $\underline{\operatorname{dim}} V:=\operatorname{dim} V^{i} v^{-i} \in \mathbb{N}\left[v^{ \pm 1}\right]$ for a finite-dimensional graded vector space $V$.

It is known [NO, Corollary 9.6.7, Theorem 9.6.8] that every simple module over $H_{k} /\left(A_{k}^{+}\right) H_{k}$ admits a grading 3 and this grading is unique up to isomorphism and shifts. By the Krull-Schmidt property every graded simple module $L$ admits a projective cover $P_{L}$. If we fix a choice $L_{1}, \ldots, L_{m}$ of graded simple modules and let $P_{1}, \ldots, P_{m}$ be their projective covers, then the classes $\left[L_{1}\right], \ldots,\left[L_{m}\right]$ (resp. $\left[P_{1}\right], \ldots,\left[P_{m}\right]$ give a free $\mathbb{Z}\left[v^{ \pm 1}\right]$-basis for $\left[\operatorname{Rep} H_{k}\right]$ (resp. [Proj $\left.H_{k}\right]$ ). Moreover, these bases are dual under $\langle-,-\rangle$.

2.3. Decomposition maps. Given any $M \in \operatorname{Rep}^{f} H_{\mathbb{K}}$ one can always find an $H_{\mathbb{O}^{-}}$ stable lattice $M_{\mathbb{O}} \subset M$ by applying $H_{\mathbb{O}}$ to a set of generators for $M$. One can argue as in $\left[\mathrm{Se}\right.$, Part III] that the class $\left[\mathbb{F} \otimes_{\mathbb{O}} M_{\mathbb{O}}\right]$ in the Grothendieck group of $\operatorname{Rep}^{f} H_{\mathbb{F}}$ does not depend on the choice of lattice. In this way one obtains the decomposition map between the Grothendieck groups:

$$
d:\left[\operatorname{Rep}^{f}\left(H_{\mathbb{K}}\right)\right] \rightarrow\left[\operatorname{Rep}^{f}\left(H_{\mathbb{F}}\right)\right] .
$$

One the other hand, standard arguments (e.g. [F] Theorem 12.3]) show that idempotents in $H_{\mathbb{F}}$ lift to $H_{\mathbb{O}}$. It follows that given any projective module $P_{\mathbb{F}}$ over $H_{\mathbb{F}}$ there exists a projective module $P_{\mathbb{O}}$ over $H_{\mathbb{O}}$ such that $\mathbb{F} \otimes_{\mathbb{O}} P_{\mathbb{O}} \cong P_{\mathbb{F}}$. Moreover, $P_{\mathbb{O}}$ is unique up to (non-unique) isomorphism by Nakayama's lemma. This process gives us the extension map:

$$
\begin{gathered}
e:\left[\operatorname{Proj}\left(H_{\mathbb{F}}\right)\right] \rightarrow\left[\operatorname{Proj}\left(H_{\mathbb{K}}\right)\right], \\
{\left[P_{\mathbb{F}}\right] \mapsto\left[\mathbb{K} \otimes_{\mathbb{O}} P_{\mathbb{O}}\right] .}
\end{gathered}
$$

2.4. Brauer reciprocity. One way of phrasing Brauer reciprocity is that $d$ and $e$ are adjoint with respect to the canonical pairing (the proof is identical to [Se, Part III]):

\footnotetext{
${ }^{3}$ Here is an intuitive explanation of this fact, which was explained to me by Ivan Losev. Let $C$ be a finite-dimensional graded algebra. The grading on $C$ is equivalent to an action of the multiplicative group on $C$. A module is gradable if and only if its twist by any element of the multiplicative group yields an isomorphic module. Now twisting preserves simple modules and their moduli are discrete (because $C$ is finite-dimensional). Hence they are fixed, and hence gradable.
} 
Lemma 2.1. We have

$$
\langle e([P]),[L]\rangle=\langle[P], d([L])\rangle .
$$

for all $P \in \operatorname{Proj} H_{\mathbb{F}}$ and $L \in \operatorname{Rep}^{f} H_{\mathbb{K}}$.

Equivalently, the matrices (with entries in $\mathbb{Z}\left[v^{ \pm 1}\right]$ ) for $d$ and $e$ in the basis $\left\{\left[L_{i}\right]\right\}_{i=1}^{m}$ and $\left\{\left[P_{i}\right]\right\}_{i=1}^{m}$ are transposes of one another.

\section{QUIVER VARIETIES AND PARITY SHEAVES}

In this section we recall briefly the definition of moduli spaces of quiver representations and explain why it makes sense to study parity sheaves on these spaces. Using a result of Maksimau [M] we then explain why describing the characters of parity sheaves on these spaces is equivalent to describing the indecomposable projective modules over Khovanov-Lauda-Rouquier algebras.

With the (possible) exception of $\$ 3.6$ and $\$ 3.7$ the material in this section is standard. The material concerning constructible sheaves on moduli of quiver representations is due to Lusztig [L2, L3] (see [Sch] for an excellent survey). For the relation to KLR algebras see $\mathrm{VV}, \mathrm{R} 2, \mathrm{M}]$.

3.1. Moduli of quiver representations. Let $\Gamma$ denote a quiver with vertex set $I$. Recall that a representation of $\Gamma$ is an $I$-graded vector space $V=\bigoplus V_{i}$ together with linear maps $V_{i} \rightarrow V_{j}$ for each arrow $i \rightarrow j$ of $Q$. Let $\operatorname{Rep} Q$ denote the abelian category of complex representations of $\Gamma$. A dimension vector is an element of the monoid $\mathbb{N}[I]$ of formal $\mathbb{N}$-linear combination of the elements of $I$. Given an $I$-graded vector space $V$ its dimension vector is $\operatorname{dim} V=\sum\left(\operatorname{dim} V_{i}\right) i \in \mathbb{N}[I]$.

Fix a dimension vector $d=\sum d_{i} i \in \mathbb{N}[I]$ and a complex $I$-graded vector space $V$ with $\operatorname{dim} V=d$. Consider the space

$$
E_{V}:=\prod_{i \rightarrow j} \operatorname{Hom}\left(V_{i}, V_{j}\right),
$$

where the product is over all arrows $i \rightarrow j$ in $Q$. Then $E_{V}$ is the space of all representations of the quiver $Q$ of dimension vector $d$ together with fixed isomorphism with $V_{i}$ at each vertex $i \in I$. If we let

$$
G_{V}:=\prod_{i \in I} G L\left(V_{i}\right)
$$

be the group of grading preserving automorphisms of $V$, then $G_{V}$ acts on $E_{V}$ by $\left(g_{i}\right) \cdot\left(\alpha_{i \rightarrow j}\right):=\left(g_{j} \alpha_{i \rightarrow j} g_{i}^{-1}\right)$. The points of the quotient space $G_{d} \backslash E_{V}$ correspond to isomorphism classes of representations of $Q$ of dimension vector $d$.

3.2. Flags and proper maps. Let us fix a dimension vector $d \in \mathbb{N}[I]$ and a sequence $\mathbf{i}=\left(i_{1}, \ldots, i_{m}\right) \in I^{m}$ such that $\sum i_{j}=d$. Given a representation $W$ with $\underline{\operatorname{dim}} W=d$, a flag on $W$ of type $\mathbf{i}$ is a flag

$$
W^{\bullet}=\left(0 \subset W^{1} \subset \ldots W^{m}=W\right)
$$

of subrepresentations of $W$ such that $W^{j} / W^{j-1}$ is isomorphic to the simple representation concentrated at the vertex $i_{j}$ with all arrows identically zero, for all $1 \leq j \leq m$. Consider the space

$$
E_{V}(\mathbf{i}):=\left\{\left(W^{\bullet}, W\right) \mid W \in E_{V} \text { and } W^{\bullet} \text { is a flag on } W \text { of type } \mathbf{i}\right\} .
$$


There is a natural embedding of $E_{V}(\mathbf{i})$ into a product of $E_{V}$ and a product of partial flag varieties, and so $E_{V}(\mathbf{i})$ has the structure of a complex algebraic variety. One may define a $G_{V}$-action on $E_{V}(\mathbf{i})$ via $g \cdot\left(W^{\bullet}, W\right):=\left(g W^{\bullet}, g \cdot W\right)$. Consider the natural projection

$$
\pi_{\mathbf{i}}: E_{V}(\mathbf{i}) \rightarrow E_{V}
$$

Then $\pi$ is clearly proper and $G_{V}$-equivariant. It is also not too difficult to see that $E_{V}(\mathbf{i}, \mathbf{a})$ is smooth.

Example 3.1. If $\Gamma$ consists of a single vertex $i$ and a single loop and $\mathbf{i}=(i, i, \ldots, i)$, then the image of

$$
\pi_{\mathbf{i}}: E_{V}(\mathbf{i}) \rightarrow E_{V}
$$

consists of nilpotent endomorphisms and $\pi_{\mathbf{i}}$ coincides with the Springer resolution of the nilpotent cone in $E_{V}=\operatorname{End}(V)$.

3.3. Constructible sheaves and KLR algebras. Fix a dimension vector $d$ and an $I$-graded vector space $V$ with $\underline{\operatorname{dim}} V=d$. In what follows we wish to consider constructible sheaves on $G_{V} \backslash E_{V}$. However, as this space is usually poorly behaved, we instead consider the $G_{V}$-equivariant geometry of the space $E_{V}$. Alternatively, we could (and probably should) consider the quotient stack $\left[G_{V} \backslash E_{V}\right]$ as in the elegant treatment of Rouquier R2]. The difference is essentially aesthetic.

Let us fix a commutative ring of coefficients $\mathbb{k}$ and consider $D_{d}:=D_{G_{V}}^{b}\left(E_{V} ; \mathbb{k}\right)$ the $G_{V}$-equivariant bounded constructible derived category of $E_{V}$ with coefficients in $\mathbb{k}[\mathrm{BL}$.

Set $\operatorname{Seq}(d):=\left\{\left(i_{1}, \ldots, i_{\mathbb{k}}\right) \in I^{k} \mid \sum i_{j}=d\right\}$. Given any $\mathbf{i} \in \operatorname{Seq}(d)$ we can consider the proper map

$$
\pi_{\mathbf{i}}: E_{V}(\mathbf{i}) \rightarrow E_{V}
$$

defined in the previous subsection. The direct image $\mathcal{L}_{\mathbf{i}, \mathbb{k}}:=\pi_{\mathbf{i} ! \mathbb{K}}$ of the equivariant constant sheaf on $E_{V}(\mathbf{i})$ in $D_{G_{V}}^{b}\left(E_{V}(\mathbf{i}), \mathbb{k}\right)$ is called a Lusztig sheaf. We set

$$
\mathcal{L}_{V, \mathbb{k}}:=\bigoplus_{\mathbf{i} \in \operatorname{Seq}(d)} \mathcal{L}_{\mathbf{i}, \mathbb{k}} \in D_{d}
$$

To $d$ we may also associate a Khovanov-Lauda-Rouquier algebra $R(d)$ (see $\mathrm{KL}$, R2 2). It is a graded algebra with idempotents $e(\mathbf{i})$ corresponding to each $\mathbf{i} \in \operatorname{Seq}(d)$. It is free over $\mathbb{Z}$ and we denote by $R(d)_{\mathbb{k}}$ the algebra obtained by extension of scalars to our ring $\mathbb{k}$.

The following result explains the relation between Khovanov-Lauda-Rouquier algebras and the geometry of the moduli space of quiver representations:

Theorem 3.2. One has an isomorphism of graded rings

$$
R(d)_{\mathbb{k}} \cong \operatorname{Ext}_{D_{d}}^{\bullet}\left(\mathcal{L}_{V, \mathbb{k}}\right) .
$$

Under this isomorphism e(i) is mapped to the projection to $\mathcal{L}_{\mathbf{i}, \mathbb{k}}$.

Proof. If $\mathbb{k}$ is a field of characteristic zero this is proved in [VV] and [R2, § 5]. For an arbitrary ring $\mathbb{k}$ this is $[\mathrm{M}$, Theorem 2.5$]$. 
3.4. Lusztig sheaves and projective modules. It is well-known and easily proved that given an object $X$ in a Karoubian additive category, then $\operatorname{Hom}(-, X)$ gives an equivalence

$$
\langle X\rangle_{\oplus}^{o p} \stackrel{\sim}{\rightarrow} \operatorname{proj} E,
$$

where $E:=\operatorname{End}(X),\langle X\rangle_{\oplus}^{o p}$ denotes the opposite category of the full additive Karoubian subcategory generated by $X$, and proj denotes the category of finitely generated projective modules over $E$ (see e.g. $[\mathrm{Kr}, \S 1.5]$ ).

One can extend this observation to graded categories (i.e., categories equipped with a self-equivalence $M \mapsto T M)$. If $X$ is an object in a graded Karoubian additive category, then $\bigoplus_{m \in \mathbb{Z}} \operatorname{Hom}\left(-, T^{m} X\right)$ gives an equivalence of graded categories

$$
\langle X\rangle_{T, \oplus}^{o p} \stackrel{\sim}{\rightarrow} \operatorname{Proj} E
$$

where $\langle X\rangle_{T, \oplus}^{o p}$ denotes the opposite category of the full additive Karoubian subcategory generated by $T^{m} X$ for all $m \in \mathbb{Z}$, and $E$ denotes $\bigoplus_{m \in \mathbb{Z}} \operatorname{Hom}\left(X, T^{m} X\right)$, a naturally graded algebra 4 and Proj $E$ denotes the graded category of finitely generated projective modules over $E$ (viewed as a graded category with self-equivalence $M \mapsto M(1))$.

Let us forget the triangulated structure on $D_{d}$ and view it simply as a graded additive category, with self-equivalence given by $\mathcal{F} \mapsto \mathcal{F}[1]$. If we apply the above observations together with Theorem 3.2 (with $V$ and $d$ as in the previous section) we see that $\operatorname{Hom}^{\bullet}\left(-, \mathcal{L}_{V, \mathbb{k}}\right)$ yields an equivalence

$$
\left\langle\mathcal{L}_{V, \mathbb{k}}\right\rangle_{[1], \oplus}^{o p} \stackrel{\sim}{\rightarrow} \operatorname{Proj} R(d)_{\mathbb{k}} .
$$

Moreover, it follows from Maksimau's proof of Theorem 3.2 that the above equivalence is compatible with an extension of scalars.

3.5. Moduli of representations of Dynkin quivers. We say that $Q$ is a Dynkin quiver if the graph underlying $Q$ is a simply-laced Dynkin diagram. If $Q$ is a Dynkin quiver we identify $I$ with the simple roots of the corresponding simply-laced root system and write $R^{+} \subset \mathbb{N}[I]$ for the positive roots. Recall Gabriel's theorem (see e.g. [B]): a quiver has finitely many indecomposable representations if and only if the underlying graph of $Q$ is a Dynkin diagram, in which case we have a bijection:

$$
\underline{\operatorname{dim}}:\left\{\begin{array}{c}
\text { indececomposable } \\
\text { representations of } Q
\end{array}\right\}_{/ \cong} \stackrel{\sim}{\rightarrow} R^{+} .
$$

Given a positive root $\alpha \in R^{+}$we denote by $I_{\alpha}$ the corresponding indecomposable representation, which is well-defined up to isomorphism.

Recall that orbits of $G_{V}$ on $E_{V}$ correspond to isomorphism classes of representations of $Q$. Hence $G_{V}$ has finitely many orbits on $E_{V}$ if and only if there are finitely many isomorphism classes of representations of $Q$ with dimension vector $d$. By Gabriel's theorem, this is the case for all dimension vectors if and only if $\Gamma$ is a Dynkin quiver.

From now on let us assume that $Q$ is a Dynkin quiver and fix a dimension vector $d$. By Gabriel's theorem and the Krull-Schmidt theorem we see that $G_{V}$-orbits on $E_{V}$ are classified by tuples:

$$
\Lambda_{d}:=\left\{\lambda=\left(\lambda_{\alpha}\right)_{\alpha \in R^{+}} \mid \sum \lambda_{\alpha} \alpha=d\right\}
$$

\footnotetext{
${ }^{4}$ Given $f: X \rightarrow T^{m} X$ and $g: X \rightarrow T^{n} X$ their product is given by $T^{n} f \circ g: X \rightarrow T^{m+n} X$.
} 
( $\lambda_{\alpha}$ gives the multiplicity of the indecomposable representation $I_{\alpha}$ as a direct summand of the isomorphism class of representation). Rephrasing this we have a stratification of $E_{V}$ by $G_{V}$-orbits

$$
E_{V}=\bigsqcup_{\lambda \in \Lambda_{d}} X_{\lambda}
$$

where $X_{\lambda}:=\left\{W \in E_{V} \mid W \cong \bigoplus_{\alpha \in R^{+}} I_{\alpha}^{\oplus \lambda_{\alpha}}\right.$ in $\left.\operatorname{Rep} Q\right\}$.

3.6. Parity sheaves on quiver moduli. We now recall the notion of a parity complex and sheaf [JMW]. As above we assume that $Q$ is a Dynkin quiver and fix the stratification

$$
E_{V}=\bigsqcup_{\lambda \in \Lambda_{d}} X_{\lambda}
$$

of $E_{V}$ by $G_{V}$-orbits. Given any $\lambda \in \Lambda_{d}$ we denote by $i_{\lambda}: X_{\lambda} \hookrightarrow E_{V}$ the inclusion of $X_{\lambda}$. Let $? \in\{!, *\}$. We say that a complex $\mathcal{F} \in D_{d}$ is ?-even if the cohomology sheaves of $i_{\lambda}^{?} \mathcal{F}$ are local systems of free $\mathbb{k}$-modules which vanish in odd degrees. We say that a complex $\mathcal{F} \in D_{d}$ is even if it is both $*$ - and !-even. We say that $\mathcal{F}$ is parity if it admits a decomposition $\mathcal{F} \cong \mathcal{F}_{0} \oplus \mathcal{F}_{1}$ with both $\mathcal{F}_{0}$ and $\mathcal{F}[1]$ even. A complex $\mathcal{F}$ is a parity sheaf if it is parity, self-dual and indecomposable.

Now suppose that $\mathbb{k}$ is a complete local ring, so that $D_{d}$ is a Krull-Schmidt category (see [JMW, $\S 2.1]$ ). We have the following classification result:

Theorem 3.3. For all $\lambda \in \Lambda_{d}$ there exists up to isomorphism at most one parity sheaf $\mathcal{E}(\lambda, \mathbb{k})$ with $\operatorname{supp} \mathcal{E}(\lambda, \mathbb{k})=\overline{X_{\lambda}}$.

Proof. The theorem is immediate from the theory of parity sheaves, once we have established that our stratified $G_{V}$-variety $E_{V}$ satisfies [JMW, (2.1) and (2.2)] and that all $G_{V}$-equivariant local systems on $X_{\lambda}$ are constant. Both of these statements hold by [M, Corollary 2.11] and [M, Lemma 3.6].

For a general Dynkin quiver one does not know if Lusztig sheaves are parity (see [M. Conjecture 1.3]). The problem is that one does not know if the fibres of the maps $\pi_{\mathbf{i}}$ have vanishing odd cohomology. If $Q$ is of type $A$, then this has been established by Maksimau (see [M, Corollary 3.36]):

Theorem 3.4. Suppose that $Q$ is of type $A$. Then for all $\lambda \in \Lambda_{d}$ there exists a parity sheaf $\mathcal{E}(\lambda, \mathbb{k})$ with $\operatorname{supp} \mathcal{E}(\lambda, \mathbb{k})=\overline{X_{\lambda}}$. Moreover, $\mathcal{L}_{V, \mathbb{k}}$ is parity and for all $\lambda \in \Lambda_{d}$, a shift of $\mathcal{E}(\lambda, \mathbb{k})$ occurs as a direct summand of $\mathcal{L}_{V, \mathbb{k}}$.

Remark 3.5. Actually, Maksimau establishes the existence of parity sheaves for any Dynkin quiver. Surprisingly, it seems difficult to show that they occur as summands of Lusztig sheaves.

3.7. Parity sheaves and the Kleshchev-Ram conjecture. In this section we assume that $Q$ is a Dynkin quiver of type $A$. We fix a dimension vector $d$. Recall our $p$-modular system $(\mathbb{K}, \mathbb{O}, \mathbb{F})$ from $\$ 2.1$.

Recall that the KLR algebra $R(d)$ is a graded ring, with each graded component free and finite rank over $\mathbb{Z}$. Moreover, $R(d)$ contains a polynomial subring and the symmetric polynomials yield a subring $A \subset R(d)$ in the centre of $R(d)$. In fact, $A$ is equal to the centre of $R(d)$ [KL, Theorem 2.9]. Finally, $R(d)$ is free of finite rank over $A$ by $\left[\mathrm{KL}\right.$, Corollary 2.10]. Hence if we set $H_{\mathbb{O}}:=R(d) \supset A_{\mathbb{O}}$, then $H_{\mathbb{O}}$ satisfies the conditions of $\$ 2.1$. 
On the other hand, if we apply the observations in 3.4 for $\mathbb{k} \in\{\mathbb{K}, \mathbb{O}, \mathbb{F}\}$ we obtain an equivalence of graded categories

$$
\left\langle\mathcal{L}_{V, \mathbb{k}}\right\rangle_{[1], \oplus}^{o p} \stackrel{\sim}{\rightarrow} \operatorname{Proj} R(d)_{\mathbb{k}} .
$$

By Theorem 3.4 of Maksimau [M] the indecomposable summands of $\mathcal{L}_{V, \mathrm{k}}$ coincide (up to shifts) with the parity sheaves. We conclude that the indecomposable graded projective modules (and hence also the graded simple modules) are parametrised up to shift by $\Lambda_{d}$.

Remark 3.6. This fact has been established algebraically for any Dynkin quiver by Kleshchev and Ram $\mathrm{KR}$.

The following is the main result of this section:

Theorem 3.7. The following are equivalent:

(1) The Kleshchev-Ram conjecture holds for $R(d)$ : the decomposition map

$$
\left[\operatorname{Rep}^{f} R(d)_{\mathbb{K}}\right] \rightarrow\left[\operatorname{Rep}^{f} R(d)_{\mathbb{F}}\right]
$$

is trivial.

(2) For all $\lambda \in \Lambda_{d}$ the parity complex $\mathcal{E}(\lambda, \mathbb{O}) \otimes_{\mathbb{Z}_{p}}^{L} \mathbb{K}$ is indecomposable.

(3) For all $\lambda \in \Lambda_{d}$ the stalks and costalks of the intersection cohomology complex $\operatorname{IC}\left(\overline{X_{\lambda}}, \mathbb{O}\right)$ are free of $p$-torsion.

Proof. By Brauer reciprocity (Lemma 2.1) statement (1) is equivalent to the extension map

$$
e:\left[\operatorname{Proj} R(d)_{\mathbb{F}}\right] \rightarrow\left[\operatorname{Proj} R(d)_{\mathbb{K}}\right]
$$

being the identity. Now the equivalence of (1) and (2) follows from the fact that (3.1) is compatible with extension of scalars.

It remains to show the equivalence of (2) and (3).

$(2) \Rightarrow(3):$ If $\mathbb{k}=\mathbb{K}$, then we can apply the decomposition theorem BBD] to conclude that $\mathcal{L}_{V, \mathbb{k}}$ is isomorphic to a direct sum of shifts of intersection cohomology complexes. We conclude that $\mathcal{E}(\lambda, \mathbb{K}) \cong \mathbf{I C}\left(\overline{X_{\lambda}}, \mathbb{K}\right)$. By the uniqueness of parity sheaves, $\mathcal{E}(\lambda, \mathbb{O}) \otimes_{\mathbb{O}} \mathbb{K}$ is indecomposable if and only if $\mathcal{E}(\lambda, \mathbb{O}) \otimes_{\mathbb{O}} \mathbb{K} \cong \mathbf{I C}\left(\overline{X_{\lambda}}, \mathbb{K}\right)$.

Let $\mathcal{E}$ be a complex of sheaves of $\mathbb{O}$-modules with torsion free stalks and costalks. Then $\mathcal{E} \otimes_{\mathbb{O}} \mathbb{K} \cong \mathbf{I C}\left(\overline{X_{\lambda}}, \mathbb{K}\right)$ if and only if $\mathcal{E} \cong \mathbf{I C}\left(\overline{X_{\lambda}}, \mathbb{O}\right)$, as follows from the characterisation of $\mathbf{I C}\left(\overline{X_{\lambda}}, \mathbb{O}\right)$ in terms of stalks and costalks BBD, Proposition 2.1 .9 and $\S 3.3]$.

Putting these two observations together, we conclude that $\mathcal{E}(\lambda, \mathbb{O}) \otimes_{\mathbb{O}} \mathbb{K}$ is indecomposable if and only if $\mathcal{E}(\lambda, \mathbb{O}) \cong \mathbf{I C}\left(\overline{X_{\lambda}}, \mathbb{O}\right)$. Hence (2) implies (3), because the stalks and costalks of $\mathcal{E}(\lambda, \mathbb{O})$ are free of $p$-torsion by definition.

$(3) \Rightarrow(2)$ : If $\mathbf{I C}\left(\overline{X_{\lambda}}, \mathbb{O}\right)$ has torsion free stalks and costalks, then it is parity (because $\mathbf{I C}\left(\overline{X_{\lambda}}, \mathbb{O}\right) \otimes_{\mathbb{O}} \mathbb{K} \cong \mathbf{I C}\left(\overline{X_{\lambda}}, \mathbb{K}\right)$ is $)$. Hence $\mathbf{I C}\left(\overline{X_{\lambda}}, \mathbb{O}\right) \cong \mathcal{E}(\lambda, \mathbb{O})$ and $\mathcal{E}(\lambda, \mathbb{O}) \otimes_{\mathbb{O}} \mathbb{K} \cong \mathbf{I C}\left(\overline{X_{\lambda}}, \mathbb{O}\right) \otimes_{\mathbb{O}} \mathbb{K}$ is indecomposable. Hence (3) implies (2).

Remark 3.8. The advantage of condition (3) in the above theorem is that it is a purely topological condition, and hence we can use it to move the Kleshchev-Ram conjecture to a question about equivalent singularities in the flag variety, where more is known and calculations are easier.

Remark 3.9. See [WB, Proposition 3.11] for results along similar lines to the above theorem. 


\section{Counterexamples}

In this section we assemble some known results due to Braden and Polo and use Theorem 3.7 to give counterexamples to the Kleshchev-Ram conjecture for quivers of type $A$.

4.1. Quiver varieties and flag varieties. We briefly recall a construction (which I learned from [KS, §8.1]) which relates an open subvariety inside the moduli of representations of a type $A$ quiver to singularities of Schubert varieties in a flag variety of type $A$.

Fix $n \geq 0$ and consider the quiver $Q$ of type $A_{2 n-1}$ with the following orientation:

$$
1 \rightarrow 2 \rightarrow \ldots \rightarrow n \leftarrow n+1 \leftarrow \ldots \leftarrow 2 n-1 .
$$

Consider the dimension vector $d=(1,2, \ldots, n, \ldots, 2,1)$. Let $I$ denote the vertices of $Q$ and let $V$ denote an $I$-graded vector space with dimension vector $d$. Inside $E_{V}$ consider the open subvariety $U$ consisting of representations all of whose arrows are injective. The product of the automorphisms of $V_{i}$ for $i \neq n$ act freely on $U$ and the quotient is isomorphic to the space of pairs on flags on $V_{n} \cong \mathbb{C}^{n}$, with the natural action of $G=G L\left(V_{n}\right)$.

Hence, after fixing a Borel subgroup $B \subset G$, the singularities of the closures of $G_{V}$-orbits on $U$ are equivalent to the singularities of closures of $G$ orbits on $G / B \times G / B$, or equivalently to the singularities of Schubert varieties in $G / B$. Combining these observations with Theorem 3.7 we deduce that if the Kleshchev-Ram conjecture holds, then stalks and costalks of all intersection cohomology complexes of Schubert varieties in $G / B$ are torsion free. The first counterexamples to this statement were given by Braden in 2004 (see the appendix to [WB]). He gave examples of 2-torsion in the costalks of intersection cohomology complexes on the flag variety of $G L_{8}$.

For several years since Braden's announcement of his results the existence of $p \neq 2$ torsion was not known. Recently, Polo $[\mathrm{P}]$ proved that for all prime numbers $p$ there exists a Schubert variety in the flag variety of $G L_{4 p}(\mathbb{C})$ whose intersection cohomology complex has $p$-torsion in its costalk. It then follows from Theorem 3.7 that the Kleshchev-Ram conjecture is false for all primes 5

The modules involved in the above counterexamples are enormous (the first counterexample involves a quiver of type $A_{15}$ !). It seems unlikely that one could verify these counterexamples algebraically, even with the help of a powerful computer. The rest of this paper is devoted to the description of the Kashiwara-Saito singularity, which occurs in a quiver of type $A_{5}$, and is small enough that one (i.e. Jon Brundan) can verify algebraically that it provides a counterexample.

4.2. The Kashiwara-Saito singularity. Let $Q$ denote the $A_{5}$ quiver:

$$
Q=1 \rightarrow 2 \rightarrow 3 \rightarrow 4 \rightarrow 5 .
$$

We identify the vertices $I$ of $Q$ with the simple roots $\alpha_{1}, \ldots, \alpha_{5}$ of a root system $R$ of type $A_{5}$. For $1 \leq i \leq j \leq 5$ let $\alpha_{i j}:=\alpha_{i}+\cdots+\alpha_{j}$. Then the positive roots of $R$ are $\left\{\alpha_{i j} \mid 1 \leq i \leq j \leq 5\right\}$. Consider the dimension vector $d=2 \alpha_{1}+4 \alpha_{2}+4 \alpha_{3}+4 \alpha_{4}+2 \alpha_{5}$ and let $V$ be an $I$-graded vector space with $\operatorname{\operatorname {dim}} V=d$.

\footnotetext{
${ }^{5}$ The author has recently found even more torsion in flag varieties of type $A[\mathrm{~W}$.
} 
As described in 3.5. $G_{V}$ orbits on $E_{V}$ are parametrised by the set

$$
\Lambda_{d}:=\left\{\sum_{1 \leq i \leq j \leq 5} \lambda_{i j} \alpha_{i j} \mid \sum \lambda_{i j} \alpha_{i j}=d\right\} .
$$

Consider

$$
\begin{gathered}
\sigma:=\alpha_{12}+\alpha_{23}+\alpha_{34}+\alpha_{45}+\alpha_{14}+\alpha_{25} \\
\pi:=2\left(\alpha_{33}+\alpha_{12}+\alpha_{45}+\alpha_{24}\right) .
\end{gathered}
$$

Then $\pi, \sigma \in \Lambda_{d}$ and we let $X_{\pi}$ and $X_{\sigma}$ denote the corresponding $G_{V}$-orbits on $E_{V}$.

Proposition 4.1. $\mathrm{IC}\left(\overline{X_{\sigma}}, \mathbb{Z}\right)$ has 2-torsion in its costalk at any point of $X_{\pi}$.

Remark 4.2. It is a result due to Kashiwara and Saito (see [KS, Theorem 7.2.1] and the subsequent remark) that the singular support of the intersection cohomology $D$-module on $\overline{X_{\pi}}$ is equal to the union of the closures of the conormal bundles to $X_{\pi}$ and $X_{\sigma}$. In particular, it is reducible. Braden B2 confirms that the characteristic cycle is equal to the sum of the fundamental classes.

Proof. Let $M_{n}(\mathbb{C})$ denote the space of $n \times n$-complex matrices over $\mathbb{C}$. Consider the space $S$ of matrices $M_{i} \in M_{2}(\mathbb{C})$ for $i \in \mathbb{Z} / 4 \mathbb{Z}$ satisfying the two conditions

$$
\begin{aligned}
& \operatorname{rank} M_{i} \leq 1 \text { for } i \in \mathbb{Z} / 4 \mathbb{Z}, \\
& M_{i} M_{i+1}=0 \text { for } i \in \mathbb{Z} / 4 \mathbb{Z} .
\end{aligned}
$$

Clearly, $S$ is an affine variety. One can show that its dimension is 8 . We call $S$ (or more precisely the singularity of $S$ at $0 \in S$ ) the Kashiwara-Saito singularity. It is known [KS, Lemma 2.2.2] that the singularity of $\overline{X_{\sigma}}$ at $X_{\pi}$ is smoothly equivalent to the singularity of $S$ at 0 .

Let $G=G L_{8}(\mathbb{C})$ and $B$ denote the subgroup of upper triangular matrices, and identify the Weyl group of $G$ with the permutation matrices. Consider the Schubert variety $X_{x}=\overline{B x B / B}$ where $x$ is the permutation $x=62845173$. Then it is not difficult to check that the singularity of $X_{x}$ along the Schubert variety $B y B / B$ where $y=21654387$ is smoothly equivalent the singularity of $S$ at 0 (see KS, Example 8.3.1]).

Because the stalks and costalks of the intersection cohomology complexes are invariant (up to a shift) under smooth equivalence, the proposition follows once one knows that $\mathbf{I C}\left(X_{x}, \mathbb{Z}\right)$ has 2 -torsion in its costalk at $y$. This has been verified by Braden (using similar techniques to those used in the appendix to WB ), by the author (using generators and relations for Soergel bimodules [EW]) and by Polo $[\mathrm{P}$ (his examples of torsion for all primes $p$ gives this example for $p=2$ ).

By Theorem 3.7 we conclude that the projective module $P\left(\sigma, \mathbb{Z}_{2}\right)$ for $R(d)_{\mathbb{Z}_{2}}$ corresponding to $\mathcal{E}\left(\sigma, \mathbb{Z}_{2}\right)$ is decomposable when we tensor with $\mathbb{Q}_{2}$. Hence the Kleshchev-Ram conjecture fails for $R(d)$. One may show that

$$
\mathcal{E}\left(\sigma, \mathbb{Z}_{2}\right) \otimes_{\mathbb{Z}_{2}} \mathbb{Q}_{2} \cong \mathbf{I C}\left(\overline{X_{\sigma}}, \mathbb{Q}_{2}\right) \oplus \mathbf{I C}\left(\overline{X_{\pi}}, \mathbb{Q}_{2}\right) .
$$

By Brauer reciprocity it follows that the simple module for $R(d)$ indexed by $\pi$ becomes reducible when one reduces modulo 2 . In fact, in $\left[\operatorname{Rep} R(d)_{\mathbb{F}_{2}}\right]$ one has

$$
\left[L\left(\pi, \mathbb{Z}_{2}\right) \otimes_{\mathbb{Z}_{2}} \mathbb{F}_{2}\right]=\left[L\left(\pi, \mathbb{F}_{2}\right)\right]+\left[L\left(\sigma, \mathbb{F}_{2}\right)\right],
$$


where $L\left(\pi, \mathbb{Z}_{2}\right)$ denotes an integral form of the $R(d)_{\mathbb{Q}_{2}}$-module labelled by $\pi$, and $L\left(\pi, \mathbb{F}_{2}\right)$ and $\left.L\left(\sigma, \mathbb{F}_{2}\right)\right)$ denote the simple $\left[\operatorname{Rep} R(d)_{\mathbb{F}_{2}}\right]$-modules labelled by $\sigma$ and $\pi$ respectively. This has been verified by direct algebraic computations by Jon Brundan (aided by his computer) and Alexander Kleshchev (with his bare hands) and has recently become available BKM, 2.6].

\section{ACKNOWLEDGEMNTS}

I would like to thank Joe Chuang and Andrew Mathas for explaining Kleshchev and Ram's conjecture to me. I also thank Alexander Kleshchev, Ruslan Maksimau and Ulrich Thiel for useful discussions, and Carl Mautner for comments on a previous version. I am very grateful to Jon Brundan and Alexander Kleshchev for finally giving algebraic confirmation of the counterexample. Finally, thanks to the referee for a careful reading and useful comments.

\section{REFERENCES}

[A] Susumu Ariki, On the decomposition numbers of the Hecke algebra of $G(m, 1, n)$, J. Math. Kyoto Univ. 36 (1996), no. 4, 789-808. MR1443748 (98h:20012)

[BBD] A. A. Běllinson, J. Bernstein, and P. Deligne, Faisceaux pervers (French), Analysis and topology on singular spaces, I (Luminy, 1981), Astérisque, vol. 100, Soc. Math. France, Paris, 1982, pp. 5-171. MR751966 (86g:32015)

[BL] Joseph Bernstein and Valery Lunts, Equivariant sheaves and functors, Lecture Notes in Mathematics, vol. 1578, Springer-Verlag, Berlin, 1994. MR 1299527 (95k:55012)

[B2] Tom Braden, On the reducibility of characteristic varieties, Proc. Amer. Math. Soc. 130 (2002), no. 7, 2037-2043, DOI 10.1090/S0002-9939-02-06469-9. MR1896039(2003d:32036)

[B] M. Brion, Representations of quivers, Séminaires et Congrès 24-I (2012), 103-144.

[BK1] Jonathan Brundan and Alexander Kleshchev, Blocks of cyclotomic Hecke algebras and Khovanov-Lauda algebras, Invent. Math. 178 (2009), no. 3, 451-484, DOI 10.1007/s00222009-0204-8. MR2551762(2010k:20010)

[BKM] J. Brundan, A. Kleshchev, Peter J. McNamara, Homological properties of finite type Khovanov-Lauda-Rouquier algebras, preprint, arXiv:1210.6900v3.

[EW] B. Elias, G. Williamson, Soergel calculus, preprint, arXiv:1309.0865v1.

[F] Walter Feit, The representation theory of finite groups, North-Holland Mathematical Library, vol. 25, North-Holland Publishing Co., Amsterdam, 1982. MR661045 (83g:20001)

$[\mathrm{J}] \quad$ Gordon James, The decomposition matrices of $\mathrm{GL}_{n}(q)$ for $n \leq 10$, Proc. London Math. Soc. (3) 60 (1990), no. 2, 225-265, DOI 10.1112/plms/s3-60.2.225. MR 1031453 (91c:20024)

[JMW] D. Juteau, C. Mautner, G. Williamson, Parity sheaves, preprint, arXiv:0906.2994v2.

[KS] Masaki Kashiwara and Yoshihisa Saito, Geometric construction of crystal bases, Duke Math. J. 89 (1997), no. 1, 9-36, DOI 10.1215/S0012-7094-97-08902-X. MR.1458969 (99e:17025)

[KL] Mikhail Khovanov and Aaron D. Lauda, A diagrammatic approach to categorification of quantum groups. I, Represent. Theory 13 (2009), 309-347, DOI 10.1090/S1088-4165-0900346-X. MR2525917 (2010i:17023)

[K] Alexander Kleshchev, Representation theory of symmetric groups and related Hecke algebras, Bull. Amer. Math. Soc. (N.S.) 47 (2010), no. 3, 419-481, DOI 10.1090/S0273-097909-01277-4. MR2651085(2012d:20022)

[KR] Alexander Kleshchev and Arun Ram, Representations of Khovanov-Lauda-Rouquier algebras and combinatorics of Lyndon words, Math. Ann. 349 (2011), no. 4, 943-975, DOI 10.1007/s00208-010-0543-1. MR2777040 (2012b:16078)

[Kr] H. Krause, Krull-Remak-Schmidt categories and projective covers, notes available at www . math.uni-bielefeld.de/ hkrause/krs.pdf

[LLT] Alain Lascoux, Bernard Leclerc, and Jean-Yves Thibon, Hecke algebras at roots of unity and crystal bases of quantum affine algebras, Comm. Math. Phys. 181 (1996), no. 1, 205-263. MR1410572 (97k:17019) 
[L1] G. Lusztig, Modular representations and quantum groups, Classical groups and related topics (Beijing, 1987), Contemp. Math., vol. 82, Amer. Math. Soc., Providence, RI, 1989, pp. 59-77, DOI 10.1090/conm/082/982278. MR.982278 (90a:16008)

[L2] G. Lusztig, Canonical bases arising from quantized enveloping algebras, J. Amer. Math. Soc. 3 (1990), no. 2, 447-498, DOI 10.2307/1990961. MR.1035415 (90m:17023)

[L3] G. Lusztig, Quivers, perverse sheaves, and quantized enveloping algebras, J. Amer. Math. Soc. 4 (1991), no. 2, 365-421, DOI 10.2307/2939279. MR.1088333 (91m:17018)

[M] R. Maksimau, Canonical basis, KLR-algebras and parity sheaves, preprint, arXiv:1301.6261v2.

[NO] Constantin Năstăsescu and Freddy Van Oystaeyen, Methods of graded rings, Lecture Notes in Mathematics, vol. 1836, Springer-Verlag, Berlin, 2004. MR2046303 (2005d:16075)

[P] P. Polo, in preparation.

[R1] R. Rouquier, 2-Kac-Moody algebras, preprint, arXiv:0812.5023v1.

[R2] Raphaël Rouquier, Quiver Hecke algebras and 2-Lie algebras, Algebra Colloq. 19 (2012), no. 2, 359-410. MR2908731

[Sch] O. Schiffmann, Lectures on canonical and crystal bases of Hall algebras, preprint, arXiv:0910.4460.

[Se] Jean-Pierre Serre, Représentations linéaires des groupes finis (French), Hermann, Paris, 1967. MR0232867 (38 \#1190)

[VV] M. Varagnolo and E. Vasserot, Canonical bases and KLR-algebras, J. Reine Angew. Math. 659 (2011), 67-100, DOI 10.1515/CRELLE.2011.068. MR.2837011

[VW] K. Vilonen, G. Williamson, Characteristic cycles and decomposition numbers, to appear in Math. Res. Let., arXiv:1208.1198.

[WB] Geordie Williamson and Tom Braden, Modular intersection cohomology complexes on flag varieties, Math. Z. 272 (2012), no. 3-4, 697-727, DOI 10.1007/s00209-011-0955-y. MR2995137

[W] G. Willamson, Schubert calculus and torsion, preprint, arXiv:0804886.

Max-Planck-Institut für Mathematik, Vivatsgasse 7, 53111 Bonn, Germany

E-mail address: geordie@mpim-bonn.mpg.de

$U R L:$ http://people.mpim-bonn.mpg.de/geordie/ 